\title{
A safety tips system for large trucks for lane change
}

\author{
Cui $\mathrm{Yu}^{1, \mathrm{a}^{*}}$, YuPing $\mathrm{Lu}^{1, \mathrm{~b}}$ and XiaoMeng Huang ${ }^{1, \mathrm{c}}$ \\ ${ }^{1}$ School of Automobile, Chang'an University, Xi'an 710064, Shaanxi, China \\ a1121406285@qq.com, b1127409795@qq.com, c2378042854@qq.com
}

Keywords: millimeter-wave radar; TTC values; truck; lane changing

Abstract. For the problem that blind spot of large trucks is relatively big and potentially rear-end in lane change and other issues. This paper proposed a large truck lane change security tips system. The system uses millimeter-wave radar to detect the positional relationship between the truck and the vehicle at rear of truck. Determine the level of security of vehicles change lanes with different values of TTC. The system can significantly reduce the incidence of truck rear-end accident in the process of lane changing.

\section{Introduction}

Vehicle lane change is a common operation behavior in driving process. The driver is to carry out the lane change according to their own needs, it can effectively improve travel speed. At the same time, the traffic resources are fully utilized. But at present, the driver training system is not perfect, and the individual difference is bigger, which leads to the driver's quality is different, and when the vehicle lane change, the dangerous target and the position relationship between the vehicle and the vehicle can be judged.

At present, the main application of the active safety system for the other road is in the passenger car, and the technology is mature. As one of the important components of the traffic participants, the safety of the truck has been paid much attention. In addition to the more common truck lane changes the existence of security risks, truck in the acceleration lane and entered the road which the rear end accidents in high incidence area. So the development of a suitable for truck for road safety prompting device is imminent.

\section{System hardware design}

System frame construction. In this system, the relative motion relationship between the vehicle and the vehicle is detected by the millimeter wave radar detecting vehicle's rear target lane, The TTC value of real time is calculated by the steering signal to start the system, according to the moving relationship between the target vehicle and the vehicle detected by radar, According to the existing TTC values of the relative maturity, the different levels of the change of the security of the road are worked out, and the different combinations of the buzzer and LED lamps are used to change the safety of the driver. The framework of the system is shown in Figure 1.

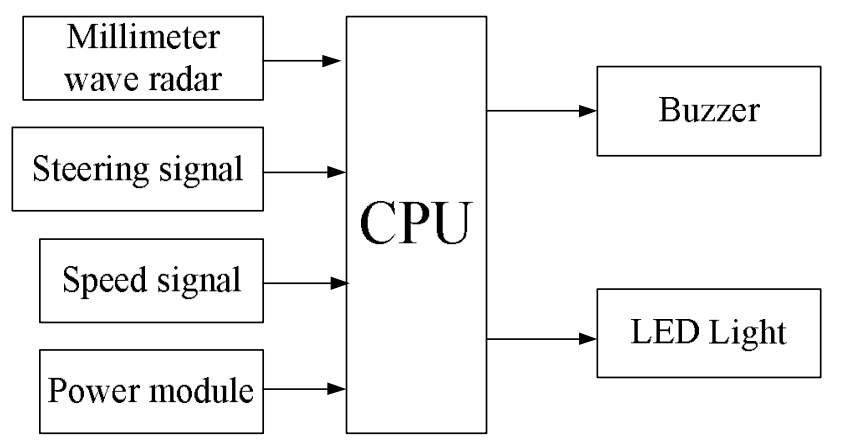

Figure 1 system frame diagram 
System signal acquisition. The system signal acquisition includes three aspects, namely, the millimeter wave radar data acquisition, the vehicle steering signal acquisition and the vehicle speed signal acquisition.

This system in millimeter wave radar using Delphi ESR millimeter wave radar, the radar has two operating modes, such as far and near, In the long distance mode, can be effectively detected within 175 meters and the car movement between plus or minus 10 degrees between the target and the car, It can be effectively detected within 60 meters and the car into a range of plus or minus 45 degrees between the target at close range. The radar can keep track of the 64 targets in the range of work, so it can meet the needs of the target vehicle data acquisition system. The vehicle steering signal is acquired by the steering signal switch to meet the requirements of low power consumption. The system will start work only when the signal lamp is turned on, and the signal is transmitted to the processor through the $\mathrm{I} / \mathrm{O}$ interface. The vehicle steering signal is acquired by the steering signal switch to meet the requirements of low power consumption. The system will start work only when the signal lamp is turned on, and the signal is transmitted to the processor through the I/O interface.

System power supply design. The car battery voltage is $24 \mathrm{~V}$, and the millimeter wave radar, the working voltage is $12 \mathrm{~V}$. The central processing unit is the MC9S12XET256 processor, which has a stable working voltage of $5 \mathrm{~V}$, LED signal lamp and buzzer are driven by $5 \mathrm{~V}$ voltage. All the equipments are powered by on-board power supply. The system uses the LM2576 to reduce the $24 \mathrm{~V}$ voltage to $12 \mathrm{~V}$ and $5 \mathrm{~V}$, which can provide a stable voltage for power supply.

Central processor design. The central processing system of the central processing unit of the MC9S12X series microcontroller, the specific model for XET256. The central processor collects the signal of I/O port in real time. When the steering lamp is turned on, the radar is launched, the moving state of the rear vehicle is detected, and the data is transmitted to the processor. The processor receives the vehicle speed signal. According to the radar data and the vehicle speed data, the TTC value is calculated according to different TTC values. The processor XET256 circuit diagram is shown in Figure 2, each pin is connected with a radar sensor, a steering signal line, a speed signal line and a tip.

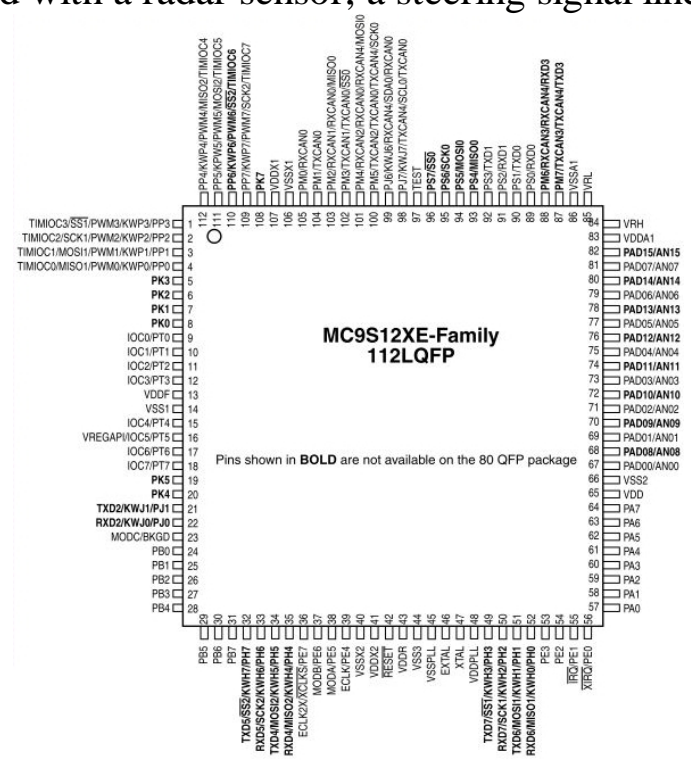

Figure 2 XET256 circuit diagram

Alarm device design. The system alarm device uses buzzer and LED lamp, according to different TTC value of the system to carry out different combinations of drivers, The LED lamp is red LED lamp, which is used for YHE12-05 Electromagnetic Active buzzer, the specific combination mode includes two kinds of single LED lamp, LED lamp and frequency buzzer.

\section{System hardware design}

The system software design is mainly to complete the safety assessment of the early warning system, and the corresponding alarm based on the assessment results. In this system, the standard of road safety 
is used to refer to the TTC value widely used in the existing system. TTC ( time to collision ) =gap/relative speed, Gap is the relative distance between the vehicle and the target vehicle, and speed relative is the relative speed of the vehicle and the target vehicle. The TTC value reflects the potential probability of collision between the vehicle and the target vehicle, but also reflects the intensity of the traffic conflict. When the relative speed of the vehicle and the target vehicle is smaller, or very near, TTC $\rightarrow \infty$, indicating that if two cars were in accordance with the current trend driving occurs the possibility of collision is almost 0 , that is to say two car is basically impossible collision. The relationship between the specific safety level and the corresponding TTC values is shown in table 1

Table 1 the relationship between TTC value and the corresponding alarm form

\begin{tabular}{|c|c|c|c|}
\hline Dangerous level & Very dangerous & General danger & Safety \\
\hline TTC value & $\leq 3 \mathrm{~S}$ & $3<\mathrm{TTC}<5.5$ & $\geq 5.5$ \\
\hline Alarm form & LED light and buzzer & LED is light & No warning \\
\hline
\end{tabular}

According to the security level, TTC value and the corresponding alarm form of Table 1, according to the data collected by the sensor and TTC, the TTC value is calculated in real time, and the TTC value and safety level are compared. The detailed flow chart is shown in Figure 3.

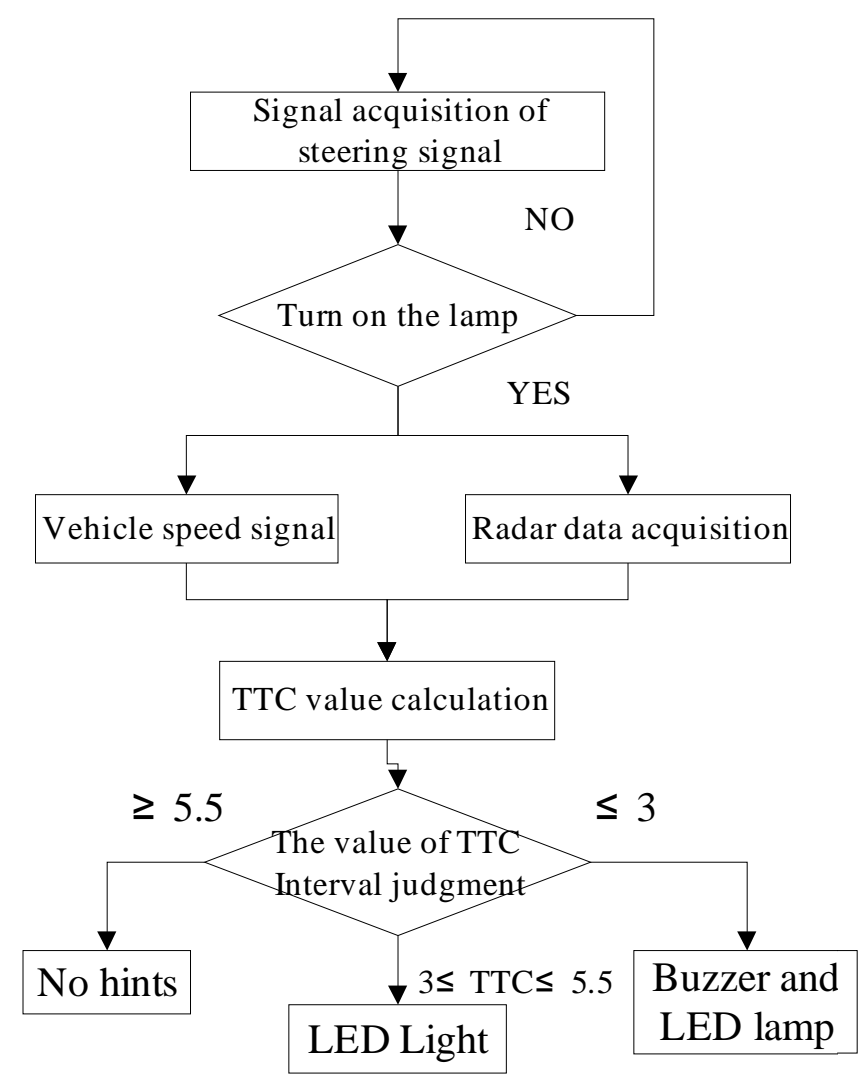

Figure 3 system work flow chart

\section{System verification}

After the system hardware and software are built, the system is used to verify the practicability of the system, The selected scene is as follows: the driver turns on the left to the left to change the road to the left, the target lane is a silver vehicle. Table 2. The radar data and the corresponding TTC values measured in a time section of the test procedure, the speed of the target vehicle is significantly higher than that of the vehicle. 
Table 2 validation scenarios and data

\begin{tabular}{ccccc}
\hline $\begin{array}{c}\text { Sampling } \\
\text { time }\end{array}$ & $\begin{array}{c}\text { Left turn } \\
\text { light }\end{array}$ & $\begin{array}{c}\text { Relative } \\
\text { distance }\end{array}$ & $\begin{array}{c}\text { Relative } \\
\text { velocity }\end{array}$ & $\begin{array}{c}\text { TTC } \\
\text { value }\end{array}$ \\
\hline $11: 28: 18$ & 1 & 15.2 & 3.47 & 4.38 \\
$11: 28: 18$ & 1 & 15.1 & 3.59 & 4.21 \\
$11: 28: 18$ & 1 & 14.9 & 3.65 & 4.08 \\
$11: 28: 18$ & 1 & 14.6 & 3.66 & 3.99 \\
$11: 28: 18$ & 1 & 14.1 & 3.63 & 3.88 \\
$11: 28: 18$ & 1 & 13.8 & 3.52 & 3.92 \\
$11: 28: 18$ & 1 & 13.5 & 3.34 & 4.04 \\
$11: 28: 18$ & 1 & 13.2 & 3.46 & 3.82 \\
$11: 28: 18$ & 1 & 12.9 & 3.39 & 3.81 \\
\hline
\end{tabular}

As can be seen from the above table, in this period of time, the vehicle is ready to change to the left lane, but its TTC value is less than the target lane between the vehicle and the road safety threshold 5.5s, Therefore, in the early warning system of the red LED lights, issued a warning warning, suggesting that at this time, there may be dangerous for the road. Therefore, in the early warning system of the red LED lights, issued a warning warning, suggesting that at this time, there may be dangerous for the road.

\section{Summary}

This paper to the existing passenger car lane change warning system based, based on millimeter wave radar and its speed design a for trucks for road safety prompting device, the device in the vehicle steering signal lamp opened the beginning of the work, the motion relationship between the data acquisition system of the car behind the target vehicle and the vehicle by real-time calculation between two vehicles of the TTC values to for road safety evaluation, results suggested that using the red LED lights and buzzer swap commented. Through the real vehicle verification system can be accurate to the freight car for the process of safety tips, with a strong practical.

\section{References}

[1] QIU Cheng-qun, LIU Cheng-lin, SHEN Fa-hua,et al. Design of automobile cruise control system based on Matlab and fuzzy PID $[\mathrm{J}]$. Transactions of the Chinese Society of Agricultural Engineering, 2012,28(6):197-202.

[2] WANG Zhi-hong, SHAO Yi-ming. Simulation of adaptive cruise contro algorithm of vehicle[J]. Computer Engineering and Design, 2014,35(2):604-608.

[3] GAO Zhen-hai, DUAN Li-fei. Modeling of Driver s Directional Control Behavior with Cerebellar Model Articulation Controller[J]. Automotive Engineering, 2012,34(4):287-291.

[4] Nadra Ben Romdhane,Mohamed Hammami,Hanene Ben-Abdallah. A Lane Detection and Tracking Method for Driver Assistance System[J].Metrol.Meas.Syst,2010,17(3):311-322.

[5] DING Neng-gen, RAN Xiao-feng, ZHANG Hong-bing. Driver Model for Single Track Vehicle Based on Single Point Preview Optimal Curvature Model[J]. Chinese Journal of Mechanical Engineering, 2008,44(11):220-223.

[6] LIU Jin-mei,LU Xue-zhu, WANG Guo-quan. Application of back propagation neural network to the orientation control of intelligent vehicle[J]. Automation \& Instrumentation, 2007,5:19-21. 